\title{
Evaluation of the Results of Treatment in Incipient Leprosy.
}

\author{
JOSE RODRIGUEZ.
}

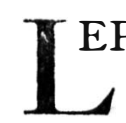

EPROSY workers are often baffled and confused by conflicting results obtained by different workers using apparently the same drugs or identical methods of treatment. One is impressed also by the long list of remedies which have been reported at one time (and these reports are usually supported by convincing statistical data and even by photographs) but which were later proven to be quite useless. The conclusion is inescapable that there is and has always been something seriously wrong somewhere about these reports.

The one serious defect common to practically all the published accounts on the treatment of leprosy so far has been the lack of proper criteria in evaluating the results of such treatment. In 1926, Acton wrote an article on the "Principles in the Testing of a Cure" (The Indian Med. Gazette, Vol. LXI, No. 6, pp. 1-26) which should be carefully read by everyone following the results of any treatment among cases of leprosy.

Admitting that the principles discussed by this authority are very difficult or may even seem to be impossible to apply in leprosy, workers in this disease must face the fact that unless these principles are applied to their work, their results will always be unreliable and subject to serious errors. The realisation of this fact is particularly important at this time when the treatment is being advocated with increasing insistence in schemes to control the disease in different countries. Anyone advocating the expenditure of large sums of money on a campaign of leprosy control or eradication, based essentially on a treatment which has not been properly controlled nor sufficiently followed to determine the permanency of the results, is assuming a very serious responsibility, to say the least.

It is not our intention to discuss in this short paper the principles already stated in the article of Lieut.-Col. Acton. We merely wish to call attention to the fact that particularly in cases of incipient or " macular " (?) leprosy it is necessary to take the utmost care in interpreting the results of the treatment, if grave errors are to be avoided.

In cutaneous leprosy, the criteria usually followed to test the efficacy of the treatment are the following :- 
1. Disappearance of acid-fast M. leprae in smears from lesions previously positive for them.

2. Disappearance in true nodules or infiltrations of the signs of activity such as redness, thickening, granular appearance, etc. One would expect that only the rash or the inexperienced would base their judgment on the disappearance of the evanescent rashes of lepra reaction, but this weakness does not seem to be at all uncommon even among old workers, judging from photographs illustrating many articles.

The above tests of a cure or of improvement in cutaneous cases are perfectly reasonable, but the usual error lies in rushing to print before the permanency of the cure or even the improvement has been determined.

When it comes to pure neural and incipient cases, the criteria followed are even more unsatisfactory, as the lesions are bacteriologically negative to begin with, and the worker is forced to judge the effects of his treatment merely on the appearance of the macules or condition of the anæsthesiawhether they are progressing, retrogressing, or stationary.

If the development of the disease were a gradual process leading from the hazy hypopigmented patches or from a group of papules to the infiltrations and nodules of the bacteriologically positive stage, it would be not so difficult to follow and to check up the results of the treatment. Unfortunately, only a small proportion of the cases follow such regular course. Usually the progress is halting and irregular. A large number show alternating but irregular periods of activation and regression during which the patient seems to be either getting worse or is improving. In a considerable number of cases with depigmented macules, the lesions remain stationary for years; suddenly there appears a crop of new lesions which rapidly become positive, or else the patient discovers that his ears are becoming infiltrated. On the other hand, such macules, after developing to a certain stage become stationary or may disappear completely and the patient becomes apparently cured.

The reddish or pink macules are even more changeable than the depigmented ones, unless there is already distinct infiltration or nodulation, in which case the lesions become stationary or progressively worse.

A more or less sudden diminution or disappearance of localised anæsthesia observed on an otherwise apparently normal-looking skin, is of ominous import among our incipient cases; there usually follows a violent efflorescence of active lesions. 
Thus, it is very easy to credit falsely apparent improvement of existing lesions to the treatment, unless the proper principles in the testing of a cure are strictly followed.

The above observations are based on a study of 336 children of leprous parents born in the Culion Leprosy Colony, most of whom had been followed personally by the writer for a period of nine years, and on 640 " incipient" or " closed" dispensary cases, 225 of whom had been repeatedly re-examined both clinically and bacteriologically for periods ranging from seven months to five years. The results of the chaulmoogra treatment among these cases are discussed in a separate paper submitted for publication in the REviEw.*

It may be of interest to mention that when those who became positive were asked to describe the type, the size, site, etc., of lesion they had when first examined, and to tell the changes in their lesions during the period of observation, it was found that only very few of them could give these details with even approximate accuracy. In most of the cases their statements were so far from the actual observed facts that the writer has come to the conclusion that studies, based on the statements of patients as to the course of the disease and the progress of the lesions, are absolutely useless. Therefore, if one wants to study the development and progress of the disease in the early stages, there is no other alternative but to observe personally a sufficient number of them for many years.

In our opinion, the only safe method of determining the efficacy of a certain preparation among these early cases is to follow closely two groups of such cases, arranged as follows :

1. Adequately treated cases.

2. Cases receiving no treatment or an inadequate number of injections.

Fortunately, it is not difficult to arrange such a grouping of cases for study. In every dispensary for the treatment of incipient cases of leprosy there is always a number of patients who report for treatment quite regularly for a period of years, while others make only a few visits a year, and not a few will not be seen again after the initial visit.

At the close of the observation period, which should not be less than five years, all the cases should be reexamined. The efficacy of the treatment may then be determined by comparing the proportion progressing to the bacteriologically positive stage among the adequately treated patients as compared with that among the insufficiently treated group.

* Published in Leprosy Review, Vol. V., No. 3, July, 1934 
Needless to say, the other precautions mentioned by Acton will have to be observed in such a study.

The nature of the disease we are dealing with is such that although it is certainly undesirable and very inconvenient, many years of observation are required before the efficacy of a certain treatment can be determined in leprosy, but this is the only safe way, particularly in the incipient stages. No short or easy methods are possible at the present time. It is precisely in this disease that the proper principles in testing a cure must be rigidly observed.

If these precautions were only followed, there would be less number of drugs so authoritatively endorsed for the treatment of leprosy, only to be finally discarded altogether or relegated to such secondary indication as control of reactions, treatment of ulcers or of eye complications, as a test to determine activity, etc.

(This article, also that which appeared in the July issue, by the same writer, is published with the approval of the Director of Health, Manila). 